Revision Article.

\title{
The Dangers of Short-Wave Diathermy
}

\author{
By MISS P. M. SCOTT, M.C.S.P., T.E.T., T.M.M.G., \\ Teacher of Physiotherapy at King's College Hospital, London. \\ Examiner for the Chartered Society of Physiotherapy. \\ Author of Clayton's Electrotherapy and Actinotherapy.
}

Short-wave diathermy is a form of electrotherapy which is used extensively, many treatments being given daily in Physiotherapy Departments throughout the world. It is only in a very small proportion of these that any untoward event occurs but it is important that this should not give a false sense of security. Accidents can happen and when they do the results are liable to be serious. Also one must consider whether the treatment is applied in such a way as to produce the best possible effects, for the value can be reduced considerably by faulty application, even if the treatment is not actually dangerous.

\section{Short-wave Diathermy Burns}

The greatest danger associated with the application of Short-wave Diathermy is that of heat burns. In the early days of the treatment it was said that deep burns could be produced with no apparent damage to the superficial tissues and though there is probably no foundation for this supposition, burns can be serious.

\section{Assessment of Intensity of Treatment}

When one considers the ways in which short-wave diathermy burns can occur it is surprising that more are not seen. The only guide as to how much current to apply is the patient's report of the intensity of heating. Consequently it is essential that the patient should understand fully the nature of the treatment and what is expected of him. He should appreciate that there is a danger of burns if the heat becomes excessive.

We are all familiar with the patient who likes a treatment to be rather uncomfortable, because then he feels that it is doing good, or who fails to report excessive heating because he "did not want to be a nuisance". In either of these cases a dangerous level of heating may be reached. Careful explanation to the patient is, however, not enough unless he is able to feel how hot the application is becoming; it is essential to test the patient's skin sensation to heat and cold before the first application and if any abnormality is present it is wiser not to apply short-wave diathermy. Some physiotherapists do give treatment under these conditions using the second electrode on some other part of the body where the sensation is normal as a control. The advisability of this is questionable for the circulatory response in the area of defective sensation will be less marked than that of the normal area. Vasodilatation results in an increased blood flow which carries heat away from the part but this will not occur to the same extent in the insensitive area as in a normal one. Consequently a higher temperature will be reached.

Clothing or any other material in contact with the tissues impairs the patient's ability to appreciate the degree of heating produced by the current, so clothing should always be removed and air spacing used whenever possible. Even if the presence of clothing does not impair the appreciation of the sensation sufficiently to be dangerous, it does reduce the efficiency of the treatment. Clothing prevents the circulation of air and the skin is heated more rapidly than when air spacing is used. Consequently the treatment feels warm and the current applied is limited before adequate heating of the deep tissues is achieved.

In order that the patient may be able to report immediately if the heating becomes excessive, he should not be allowed to fall asleep during the treatment and short-wave diathermy should not be used for patients who are mentally defective or liable to lose consciousness, as, for example, epileptics.
For the same reason children are unsuitable for this form of treatment. It is impossible to state a definite age at which a child is able to understand and co-operate enough for an effective treatment to be given. The practice of the physiotherapist keeping her hand on the area throughout the treatment is not satisfactory; it is unlikely that the patient's tissues and the physiotherpist's hand are heated to the same extent.

Having taken all possible steps to ensure that the patient understands and can appreciate the degree of heating required, the physiotherapist must be at hand during the treatment session to reduce the current immediately if heating does become excessive.

\section{Concentration of the Electric Field}

Overheating of the tissues with short-wave diathermy is most likely to occur if there is some factor which causes concentration of the electric field. This may result from one part of an electrode lying closer to the tissues than does the remainder and can occur if the electrode is not parallel to the skin or is placed with inadequate spacing over a prominence. This is an additional reason for removing clothing as its presence makes it difficult to judge the position and spacing of electrodes accurately. If a lead touches or even approaches too closely to the tissues a localized electric field is set up and overheating results. The leads should be separated from the tissues by insulating material at least as thick as the electrode spacing.

The lines of force pass more easily through some materials than others and the presence of a localized area of such material is liable to cause concentration of the field. The outstanding examples of such materials are metal and water and it is essential that there are no localized areas of these in the region of the part being treated.

\section{Presence of Metal}

Metal may be embedded in the tissues, for instance shrapnel left from an old war wound or plates or pins used for the fixation of fractures. The size of the metal and the position in which it is lying relative to the electric field determine whether treatment is dangerous or not. In some cases the current can safely be applied, but the decision must rest with the Medical Officer rather than with the physiotherapist, and be confirmed by him. Clothing may contain unexpected metal in the form of press studs, clips for adjusting shoulder straps and bracers, or suspenders, but with the removal of clothing danger from these sources is eliminated. Other metal objects such as hair clips or rings can be removed from the surface of the body.

\section{Presence of Moisture}

Localized moisture causes concentration of the electric field and widespread dampness is dangerous as the water tends to become hot and may cause a scald. This can occur in the presence of a wet dressing or if clothing or the towels used for inductothermy are damp. Even if there is no actual scald excessive sensation of warmth will limit the amount of current applied and reduce the deep heating effect. In hot weather clothing may be damp from perspiration so the area being treated should always be uncovered, and great care taken to keep short-wave diathermy equipment quite dry. 
Wounds

Open wounds need special attention as safety pins are sometimes used to secure drainage tubes and a damp dressing or discharging wound can be a source of danger. Wounds should always be cleaned and a dry dressing applied immediately before commencing treatment; if there is much discharge it may be necessary to renew the dressing during treatment.

\section{Impaired Circulation}

Overheating and consequent burning of the tissues can occur if the blood flow is impaired. Thus pressure on the area, as may occur when a patient is lying on a malleable electrode with felt spacers, or when the cable is coiled round a limb, can cause a burn. Steps should be taken to relieve the pressure on the part.

A very real danger with short-wave diathermy lies in the application of treatment to areas which have a defective arterial blood supply, such as the distal parts of limbs affected by arteriosclerosis or Buerger's disease. Heat increases metabolism and the demand for oxygen which is normally met by vasodilatation and increased flow of arterial blood into the area. Where normal dilatation of the arteries cannot take place the necessary oxygen is not supplied and death of the tissues-i.e. gangrene-may result.

\section{Overdose}

It is possible to give an overdose of short-wave diathermy with consequent aggravation of symptoms. This is most liable to occur when treating an inflammatory lesion within a confined space, such as sinusitis or a boil in the ear, but increase in pain can follow excessive treatment in other conditions. In view of the difficulty in assessing dosage it is surprising that this does not occur more frequently but probably the tendency is to err on the side of under rather than overdosage, with reduction of the efficacy of the treatment.

\section{Electric Shock}

As with any electrical treatment there must be some danger of electric shock, but this is less liable to occur with short-wave diathermy than with some other treatments. | Earth shock from the apparatus casing onto which the live wire of the main is leaking is the chief danger, but should not occur if the apparatus is maintained in good condition. It is possible to get sparks from conductors which lie within the region of the field and are consequently electrically charged, but these are unpleasant rather than dangerous.

\section{General Effects}

An extensive short-wave diathermy treatment produces effects throughout the body. As the heated blood circulates there is a general rise in temperature and the patient may catch a chill if he goes out in the cold immediately after treatment. The rise in temperature is accompanied by generalized vasodilatation which results in a fall in blood pressure. This may cause the patient to feel giddy if he rises suddenly from a recumbent position. Giddiness also occurs sometimes during treatments throughout in the region of the head. The exact reason for this, and whether short-wave diathermy has any definite effects on the brain, are not known, but it is wiser to avoid as far as possible arrangements of electrodes that pass the current through the brain.
THE BIRTCHER

\section{crusader}
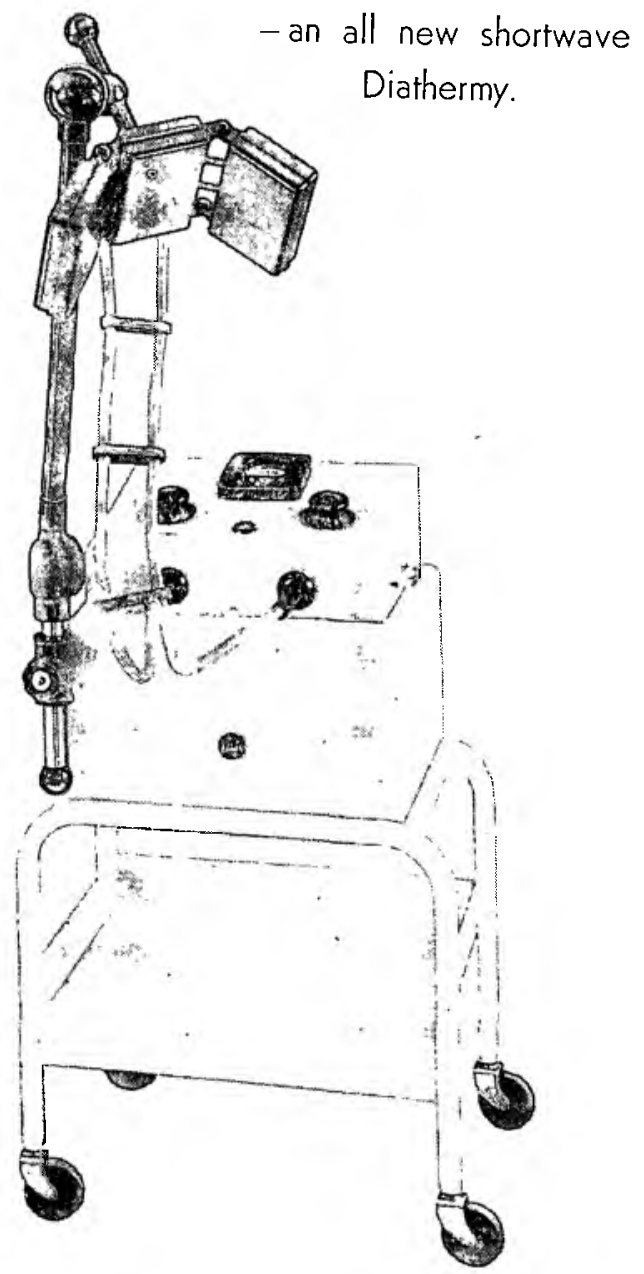

Imagine ... a genuine BIRTCHER at only $R$ 364.50*

including Standard Accessories and Trolley

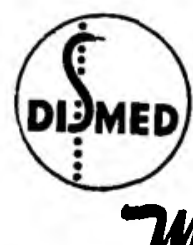

*With Triple Drum as shown in illustration R475.00 GUARANTEED FOR 2 YEARS, INCLUDING TUBES.

BUILT in accordance with NEW FREQUENCY REGULATIONS.

\section{Medical Distributous} (PTY.) LTD.

\author{
"Cape York", 252 Jeppe Street, Johannesburg \\ P.O. Box 3378 \\ Telephone 23-8106 \\ and at \\ 20 Barrack Street, Cape Town \\ P.O. Box 195 \\ Telephone 41-1172
}

